\title{
Cultura digital y nuevos perfiles profesionales: desafíos regionales ${ }^{*}$
}

Cristóbal Cobo Romaní

Research Fellow, Oxford Internet Institute at the University of Oxford

cristobal.cobo@oii.ox.ac.uk

|Fecha presentación: 16/03/2010 | Aceptación: 29/09/2010 |Publicación: 17/12/2010

\section{Resumen}

Este trabajo propone una revisión de las nuevas tendencias en el mundo del trabajo, su orientación hacia los servicios, la gestión del conocimiento y el uso de las nuevas tecnologías. Bajo este marco se analizan algunos de los desafíos que debe enfrentar la educación actual y propone un marco de análisis en relación a aquellas competencias y habilidades que contribuirían a formar a un trabajador e-competente, adaptable y en permanente aprendizaje.

Palabras claves: e-competencias, trabajador del conocimiento, nuevas tecnologías, sociedad del conocimiento, educación

\section{Resum}

Aquest treball proposa una revisió de les noves tendències en el món del treball, la seua relació amb els serveis, la gestió del coneixement i l'ús de les noves tecnologies. Baix aquest marc, s'analitzen alguns dels desafiaments que ha d'enfrontar l'educació actual i proposa un marc d'anàlisi en relació a les habilitats i competències que contribueixin a formar un treballador e-competent, adaptable i en permanent aprenentatge.

Paraules clau: e-competències, treballador del coneixement, noves tecnologies, societat del coneixement, educació

\section{Abstract}

This work proposes a review of the new trends in the world of labour, its relation to services, knowledge management and the use of new technologies. In this context, the challenge that current education faces is reviewed. The paper proposes a framework of analysis for those skills and competencies that would contribute to train an e-competent, adaptable and continuously learning worker.

Keywords: e-competencies, knowledge worker, new technologies, knowledge society, education

\footnotetext{
* Parte de los resultados de esta investigación fueron realizados gracias a la financiacón del Economic and Social Research Council (ESRC) y Social Science Research Council (SSRC). Este estudio se realizó en Centre on Skills, Knowledge and Organisational Performance (SKOPE), University of Oxford. http://www.ecompetencies.org/.
} 


\section{Introducción}

Este texto busca analizar las tendencias relacionadas con las demandas del mercado laboral de los próximos años (2010-2020) en Europa e Iberoamérica y su vínculo con el desarrollo de habilidades insertas en el contexto de la sociedad del conocimiento.

Las observaciones y tendencias aquí expuestas buscan describir los desafíos que ha de enfrentar la formación de una fuerza laboral competente y en permanente estado de actualización. La revisión documental aquí descrita está orientada a identificar pautas y criterios de utilidad para el diseño de políticas públicas tanto en educación como en formación de capital humano.

Entre los interrogantes que detonan este breve análisis destacan las siguientes preguntas: ¿Cómo afectan las nuevas demandas del mundo del trabajo a las estrategias educativas actuales? ¿La incorporación de nuevas tecnologías en los entornos de aprendizaje ha resultado suficiente para preparar mejor a quienes se incorporan al mundo del trabajo? ¿La educación está cambiando más allá de la adopción de nuevos dispositivos tecnológicos? A estos interrogantes se anteponen las palabras de Zygmunt Bauman:

"Al menos en la parte desarrollada del planeta se han dado, o están dándose ahora, una serie de novedades no carentes de consecuencias y estrechamente interrelacionadas, que crean un escenario nuevo y sin precedentes para las elecciones individuales, y que presentan una serie de retos nunca antes vistos" (Bauman 2007: 7)

Esta oportuna reflexión de Bauman ofrece un punto de partida para conceptualizar los tiempos líquidos en que vivimos, sujetos a profundas redefiniciones e incertidumbres. En el caso puntual de este documento, el análisis de estas transformaciones atenderá particularmente el binomio: educación-preparación laboral. Si bien en muchas latitudes de las sociedades del conocimiento existe un consenso de la necesidad de incorporar mejoras sustantivas en la educación, en muchos casos se observa que esas mejoras se han limitado a cambios parciales y, por sobre todo, a la adquisición de prótesis educativas, más conocidas como tecnologías digitales.

\section{Datos y análisis}

El trabajador del conocimiento: En cuanto a las transformaciones del mercado del trabajo, así como del sector productivo, estas redefiniciones comienzan a evidenciarse con significativa anterioridad al cambio de siglo. Dos casos evidentes de ello son los trabajos de Bell (1976) y Machlup (1962).

Un ejemplo clásico de esta reconceptualización del mundo laboral es el término trabajadores del conocimiento, acuñado por Drucker en 1959, el cual hace referencia a aquellos sujetos que trabajan principalmente con información o, bien, gestionan conocimiento en su empleo. Individuos que cuentan con los saberes, habilidades y destrezas para adquirir, procesar, administrar y comunicar conocimientos de manera adecuada en diferentes contextos de trabajo.

Lo interesante de la conceptualización que proponía Drucker, hecha justo hace cincuenta años, es su compren- sión de cómo el conocimiento jugaría un papel central en las actividades diarias del sector productivo y de servicios.

Indudablemente que el perfil de este trabajador del conocimiento ha evolucionado de manera simultánea a la maduración de fenómenos como: la globalización, la expansión de los mercados, la masificación de las nuevas tecnologías, la incorporación de la mujer en la fuerza productiva, la valorización de las economías orientada a los servicios, entre otros.

Fuerza laboral y competencias: El desarrollo constante de habilidades y competencias hoy resultan acciones estratégicas para poder acceder a una mejor calidad de vida y también de empleo. Sin embargo, su adquisición a menudo resulta más difícil para quienes más los necesitan: aquellos que están atrapados en empleos mal remunerados, con difíciles condiciones laborales y precarios niveles de formación.

En este marco, uno de los desafíos centrales es soslayar la brecha entre la política del mercado laboral, las demandas de empleo del sector productivo y el tipo de formación profesional que reciben los trabajadores. En otras palabras la articulación entre empleo, educación y políticas públicas.

Bajo un análisis comparativo la Organización para la Cooperación y el Desarrollo Económico ${ }^{1}$ (OECD, 2006) destaca algunos elementos centrales en la correlación entre inversión en capital humano, productividad de la fuerza laboral y el crecimiento de las naciones. Este organismo internacional destaca la falta de correspondencia entre aquellas habilidades enseñadas en las escuelas y las requeridas por las empresas, agregando que muchos países están experimentando serios vacíos y desajustes. Fenómeno que afecta directamente los niveles de empleabilidad de su fuerza de trabajo actual, así como de las décadas venideras.

En esta línea se identifica que hoy en día las naciones enfrentan dificultades para mejorar los niveles de formación de su fuerza de trabajo, particularmente de los trabajadores menos cualificados (low skilled workers). La OECD destaca que avanzar en este ámbito resulta especialmente estratégico si se toma en consideración que existe una estrecha relación entre la cualificación de la fuerza laboral y la productividad de una nación.

La Figura 1 revela cómo el tema de los nuevos perfiles profesionales que hace un uso extensivo de las nuevas tec-

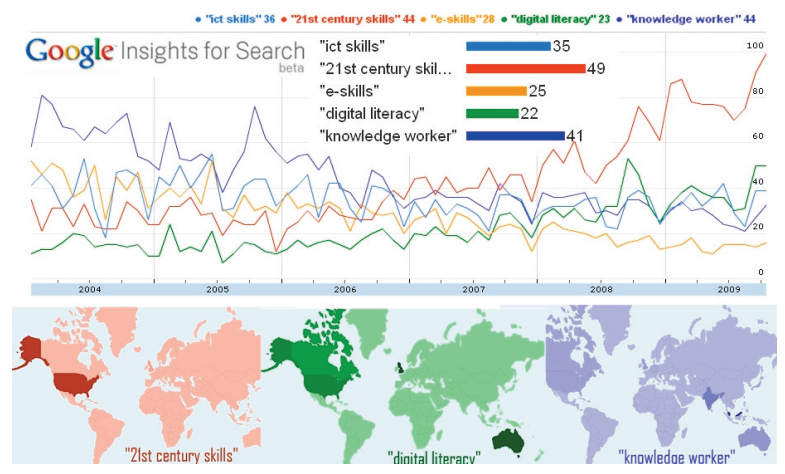

Figura 1- Google Insights: Trabajadores del Conocimiento y Globalización. Fuente: (2010 Google. Todos los derechos reservados. Se autoriza su uso para fines educativos.

\footnotetext{
${ }^{1}$ La sigla OECD proviene del anglicismo Organisation for Economic Co-operation and Development.

${ }^{2}$ Esta imagen fue elaborada por el autor al utilizar la herramienta Google Estadística de Búsqueda. Tal como lo describe el sitio oficial esta herramienta permite comparar patrones de volumen de búsqueda en determinadas regiones, categorías, intervalos de tiempo y propiedades. Consulte distintos ejemplos sobre el uso de Estadísticas de búsqueda de Google. [www.google.com/insights/].

Nota del Autor: Esta herramienta resulta pertinente para visualizar en términos sumamente generales tendencias de búsqueda. Por ello, estos datos crean un gráfico a partir del interés que generan estos términos de búsqueda concretos. Es esperable que el uso e interés por dichos conceptos varíe según el contexto o perfil del usuario.
} 
nologías y el conocimiento resulta una realidad global. De igual modo, aunque resulta ser una fotografía general (no necesariamente exhaustiva) da cuenta de cómo la adopción de conceptos estrechamente relacionados varía según la región del planeta que se observa. Por ejemplo, mientras en Estados Unidos se presta mayor énfasis a la idea de competencias para el siglo 21 (21st century skills) en Canadá o Australia se observa una mayor relevancia del concepto de alfabetismo digital (digital literacy).

Mundo Plano: A continuación se ofrece una revisión sobre tendencias que se identifican tanto en el continente europeo así como en la región iberoamericana. Estos antecedentes denotan cómo las redefiniciones del mercado productivo habrán de incidir en las estrategias educativas y de capacitación de sus respectivas naciones.

Europa

El estudio "Nuevas competencias para nuevos empleos", publicado por el European Centre for the Development of Vocational Training (CEDEFOP, 2008) sugiere que el continente europeo está experimentando un cambio general del sector primario (particularmente en la agricultura) y las industrias manufactureras tradicionales hacia los servicios y la economía de conocimiento. Ésta se plantea como una tendencia clave particularmente durante la próxima década.

En su análisis se indica que más de 20 millones de nuevos puestos de trabajo se crearán durante el periodo 2006 y 2020 en la UE-25. En esta línea, destaca el que casi las tres cuartas partes de los puestos de trabajo en la UE-25 $+{ }^{3}$ estarán insertos en el sector de servicios para el año 2020.

Indudablemente que estas proyecciones y el papel estratégico que juegan ámbitos como el uso del conocimiento, la innovación y las nuevas tecnologías contrasta con algunas de las características que hoy tiene la población europea. Llama la atención, por ejemplo, un estudio publicado por EUROSTAT (2006). Éste indica que más de un tercio (37\%) de la población europea no posee habilidades digitales (computer skills) del cual el 60\% posee un nivel educativo igual o inferior a secundaria.

De plasmarse estas proyecciones, descritas por CEDEFOP, profundas repercusiones habrán de esperarse en Europa, particularmente para los sectores industriales, educativos y de capacitación.

Por último, este análisis plantea que este fenómeno afectaría también ámbitos como la migración, la movilidad intra-Europa, las características y duración de la vida laboral, los tipos de empleos y de habilidades requeridas, entre otros aspectos afines (CEDEFOP, 2008).

América Latina/Iberoamérica

Resulta oportuno complementar estas proyecciones del panorama laboral europeo con la realidad actual de Iberoamérica. A continuación se ofrecen algunos extractos del documento "Espacios iberoamericanos: la economía del conocimiento" (CEPAL, 2008).

La actual economía del conocimiento abre nuevas oportunidades para la transformación productiva de los países iberoamericanos y plantea sustantivas desafíos en cuanto a aumentar la calidad y cantidad de recursos humanos.

Los nuevos paradigmas tecnológicos como las tecnolo- gías de información y de comunicación (TIC), la biotecnología y la nanotecnología, se han traducido en una creciente demanda de recursos humanos calificados.

Los servicios representan alrededor de dos tercios del producto interno bruto de los países desarrollados y casi la mitad de los países en desarrollo. A su vez, el sector absorbe el $70 \%$ del empleo de los primeros y un tercio de la mano de obra de las economías emergentes.

La rama de otros servicios, que incluye servicios empresariales, profesionales, de computación, información, construcción, arquitectura, ingeniería, medicina y publicidad, ha sido la de mayor dinamismo en las últimas dos décadas.

En las economías de Iberoamérica, el peso de los sectores de uso intensivo de tecnología no supera el 20\% del valor agregado generado en la industria manufacturera en su conjunto.

El gran rezago a nivel de educación universitaria de los países iberoamericanos obedece a la reducida matrícula de estudiantes en cursos universitarios. Es fundamental aumentar la masa crítica de la formación en materias científicas para satisfacer la demanda creciente de capital humano calificado.

Resulta interesante observar que, a pesar de las diferencias estructurales entre el contexto de la UE y la realidad iberoamericana, en ambos frentes existen elementos comunes. Entre las complementariedades se identifican aspectos como: el papel estratégico que ha de jugar el rubro de servicios; la importancia de contar con una fuerza laboral altamente calificada, el papel clave del conocimiento y la innovación, así como del uso estratégico de las tecnologías de información y comunicación.

Thompson, autor de El Mundo es Plano (2005) agrega que aquellos sectores de la fuerza laboral cuyo trabajo sea potencialmente reemplazable muy probablemente quedarán subsumidos bajo las fuerzas de un mercado globalizado en el cual todos son potencialmente competencia para todos.

Cualquier trabajo que pueda ser llevado a prácticas rutinarias y transformado en bits y bytes hoy puede ser exportado a otros países que ya han alcanzado un rápido crecimiento de trabajadores del conocimiento altamente capacitados que trabajarán por una pequeña fracción del salario original.

Este autor identifica algunos vectores que aceleran esta transformación, entre los que destacan dos fenómenos claves: outsourcing (subcontratar proveedores que puedan ofrecer servicios más eficientes a costos más reducidos que los desarrollados por la propia organización) y offshoring (cuando la empresa decide llevar sus plantas de producción a otro país en busca de costos más bajos).

Estas estrategias son posibilitadas, en buena medida, por el uso ubicuo y estratégico de las TIC que permiten conectar y aplanar las diferencias entre mercados, culturas y regiones.

\section{Nuevos alfabetismos}

La Estrategia de Lisboa 2000-2010 ${ }^{5}$ definida conjuntamente por los miembros de la UE en materia de crecimiento, productividad y empleo, identifica ocho competencias claves para desempeñarse en la sociedad del conocimiento: la comunicación en la lengua materna; la comunicación en lenguas extranjeras; la competencia matemática y las competencias básicas en ciencia y tecnología; la competencia digital; el aprender a aprender; las competencias sociales

\footnotetext{
${ }^{3}$ Austria, Belgium, Bulgaria, Cyprus, Czech Republic, Denmark, Estonia, Finland, France, Germany, Greece, Hungary, Ireland, Italy, Latvia, Lithuania, Luxembourg, Malta, The Netherlands, Poland, Portugal, Romania, Slovakia, Slovenia, Spain, Sweden y United Kingdom.

${ }^{4}$ Cuyo título original de esta obra es The World Is Flat: A Brief History of the Twenty-First Century, escrito por Thomas L. Friedman.

${ }^{5}$ http://europa.eu/scadplus/glossary/lisbon_strategy_es.htm.
} 
y cívicas; el sentido de la iniciativa y el espíritu de empresa; la conciencia y la expresión cultural.

Indudablemente que dichas habilidades y conocimientos no están restringidas únicamente a quienes han de emplearse en el continente europeo, sino que por el contrario, estas competencias han de ser entendidas, por ejemplo en América Latina, como pautas a considerar por quienes diseñan los programas educativos actuales ${ }^{6}$.

Una reflexión orientada a conceptualizar el perfil de profesional de quienes buscarán emplearse en este mundo plano se encuentra en el trabajo de Wagner (2008). En su investigación este autor propone siete habilidades fundamentales para enfrentar los cambios y redefiniciones de esta era globalizada. Según expone, las habilidades (soft skills) que contribuirían a una mejor formación de la fuerza laboral del siglo XXI son:

1. La solución de problemas y pensamiento crítico;

2. Colaboración a través de redes de cooperación einfluencia;

3. Agilidad y capacidad de adaptación;

4. Iniciativa y espíritu de empresa;

5. Eficacia comunicacional, escrita y oral;

6. Capacidad para acceder y analizar información, y

7. Curiosidad e imaginación.

Wagner, postula que tanto en el mundo del trabajo como en el del aprendizaje es básico desarrollar la capacidad de pensar. Es decir, razonar, analizar, ponderar evidencias y tener la habilidad para resolver problemas y para comunicarse de manera efectiva. Hoy, agrega el autor, éstas no son competencias que requieren únicamente las élites de cada país, como podría haber ocurrido en el pasado, sino que por el contrario han de ser entendidas como habilidades que todos los sectores necesitan desarrollar.

De manera complementaria, Silva plantea que el mundo del trabajo requerirá empleados que sepan cómo hacer las cosas (know how) más que entes que únicamente repliquen procedimientos. En otras palabras, sujetos que reconozcan cuál es la información relevante, por qué y fundamentalmente cómo se conecta con otras fuentes. El énfasis de esta perspectiva está en qué hacer con el conocimiento, más allá de qué unidades de conocimientos tiene cada uno (Silva, 2008).

La perspectiva del trabajador del conocimiento que describe Silva se complementa de manera interesante, además de las ideas de Drucker, con postulados del connectivismo (Siemens, 2004). Algunos de los principios de esta propuesta teórico-pedagógica impulsada por Siemens, plantean que el aprendizaje es un proceso que se enriquece a través de la conexión y vinculación de nodos o fuentes de información. En esta línea, las conexiones entre ideas y campos del conocimiento pueden detonar insospechadas innovaciones.

Siemens destaca que la capacidad de construir conexiones entre diferentes fuentes de información posibilita nuevas oportunidades de aprendizaje. Esta aproximación conceptual destaca que las tecnologías se convierten en herramientas facilitadoras de nuevos conocimientos y aprendizajes. Sin embargo, este acto de conectar saberes es una habilidad que ha de ser desarrollada por sujetos competentes, sean estos estudiantes o trabajadores.

Otro aporte, que ofrece postulados conceptuales que enriquecen lo aquí descrito, es Gilster autor del libro Alfabetismo

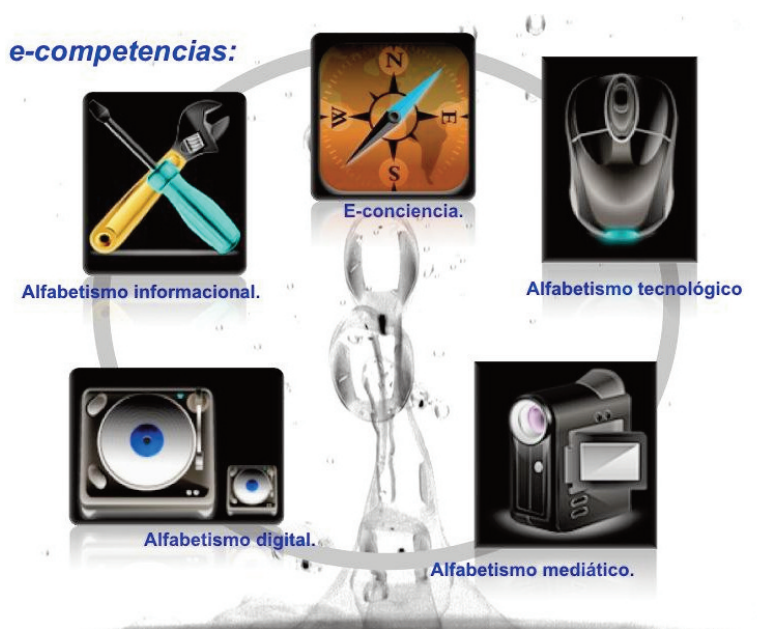

Figura 2- Meta e-competencias. Fuente: Elaboración propia.

Digital (1997). En su trabajo escribió que la alfabetización digital tiene que ver con el dominio de las ideas no de las teclas. En su reflexión subyace el interés por promover el desarrollo de nuevos alfabetismos y competencias. Este autor sugiere que la utilización de las TIC no debe limitarse al uso de una tecnología en particular. Ello puesto que la conexión de significados y el componente contextual cumplen un rol estratégico en la formación y desempeño de los trabajadores del conocimiento.

Dicho lo anterior, y a modo de contribución de este artículo, a continuación ofrecemos un aporte conceptual orientado a mapear el perfil de un trabajador del conocimiento, el cual para los términos de este análisis ha sido denominado trabajador e-competente.

Después de haber identificado distintos aportes relacionados con el perfil de un trabajador del siglo XXI, a continuación proponemos un esquema conceptual complementario (en ningún caso excluyente) que busca articular diversas fuentes académicas y/o provenientes de organismos internacionales.

Este ejercicio ha tenido como fin identificar aquellas competencias vinculadas al uso de las tecnologías digitales y la gestión del conocimiento, que pudiesen apoyar el desempeño laboral de quienes trabajan en la era de la información (más detalles consultar la siguiente dirección: tiny.cc/eskills).

Producto de esta revisión, a continuación proponemos una definición del término e-competencias:

Capacidades para la gestión de conocimiento tácito y explícito, empoderada por la utilización de las TIC y el uso estratégico de la información. Las e-competencias van más allá de la utilización de una TIC en particular, ya que también incluyen conocimientos y actitudes orientadas al trabajo colaborativo, la innovación y el aprendizaje constante, así como la creación de nuevas ideas para enfrentar problemas desconocidos en diversos contextos.

El perfil de un trabajador e-competente está constituido por cinco habilidades fundamentales: e-conciencia; alfabetismo tecnológico; alfabetismo informacional; alfabetismo digital y alfabetismo mediático. Cada una de estas se describen a continuación (Figura 2).

E-Conciencia: Esta habilidad cognitiva se caracteriza por la comprensión del papel que juegan las TIC en la

${ }^{6}$ Esta iniciativa es complementaria a otras experiencias orientadas a consolidar la formación por competencias como por ejemplo el proyecto: Alfa Tuning (http://tuning.unideusto.org/tuningal). 
sociedad del conocimiento. Está basada en el entendimiento de cómo estos instrumentos pueden resultar perjudiciales y/o benéficos para el desarrollo de la sociedad. Es un acto de cognición influenciado por los crecientes flujos de información y conocimiento orientado a la generación de valor agregado en diversos contextos. Un usuario e-conciente cuenta con la capacidad de entender y responder a los desafíos de una sociedad que asigna especial importancia a la idea de aprendizaje para toda la vida. Este entendimiento incluye la habilidad para vincular los ámbitos sociales, culturales, legales y éticos relacionados a las TIC (ciudadanía digital).

Alfabetismo Tecnológico: Este alfabetismo guarda relación con el uso diestro de los medios electrónicos tanto para estudiar y trabajar como para el ocio. Está representado por la habilidad de interactuar tanto con hardware y software así como con aplicaciones vinculadas con la productividad, la comunicación o la gestión. Este alfabetismo incluye el uso estratégico de Internet y otras vías electrónicas de comunicación para actividades como: generar de redes de colaboración, intercambio de información, trabajo a distancia, entre otras. Las tecnologías incluidas en esta competencia evolucionan de acuerdo a la transformación de las TIC.

Alfabetismo Informacional: Es la habilidad de comprender, evaluar e interpretar información proveniente de diferentes fuentes. El concepto de alfabetismo informacional va mucho más allá que la capacidad de leer, puesto que significa leer con significado, entender críticamente y al mismo tiempo ser capaz de analizar, ponderar, conectar e integrar diferentes informaciones, datos y conocimientos. Requiere la capacidad de hacer juicios informados en relación a aquello que se encuentra dentro o fuera de Internet. Incluye la capacidad de evaluar el grado de confiabilidad y calidad de la fuente, determinando cómo y cuándo la información es apropiada para una determinada audiencia o contexto.

Alfabetismo Digital: Es la capacidad para generar nuevo conocimiento apoyado en el uso estratégico de las TIC. Los principales aspectos vinculados con la alfabetización digital combinan la habilidad para conseguir información relevante (dimensión instrumental) así como producir y administrar nuevo conocimiento (dimensión estratégica). Estar alfabetizado digitalmente implica utilizar las TIC para acceder, almacenar, organizar, integrar, y compartir información y conocimiento en múltiples formatos.

Alfabetismo Mediático: Este alfabetismo tiene que ver con comprender cómo los medios de comunicación tradicionales están transformándose dentro del entorno de los medios electrónicos. Esta habilidad incluye el entendimiento de cómo funcionan los medios, cómo evolucionan hacia nuevos formatos, sus plataformas y modos de interacción. Finalmente, incluye el saber cómo los medios producen y generan significado así como sus implicancias sociales, legales, políticas y económicas.

Antes de concluir, podemos plantear que tras años creyendo que la forma de modernizar las escuelas, y la enseñanza en general, era a través de la incorporación de TIC, hoy contamos con estudios suficientes como para comprender que esta tarea incluye además (y quizá antes que la incorporación de dispositivos) elementos como: definición de competencias específicas, incorporación de nuevas prácticas pedagógicas, des-uniformar el proceso de aprendizaje, revalorizar el aprendizaje informal, rediseñar el currículum y los sistemas de evaluación, repensar la interacción escuelasociedad y universidad-empresa, entre muchos otros.

Sin duda que uno de los principales desaciertos ha sido creer que las TIC son aparatos que por sí sólos cambiarían las dinámicas nonagésimas de la educación (Buckingham, 2007, Cobo, 2009). El mito que venimos arrastrando desde hace años atrás es que lo importante es aprender a usar los aparatos porque eso es lo que nos preparará para esta sociedad del conocimiento.

Hoy cada vez son más las voces que insisten en la importancia de aprender la gramática de las tecnologías, es decir el uso de la información a través de distintos dispositivos y software, pero acompañado de un conjunto más complejo de destrezas y conocimientos, que nos ayudan a sobrellevar la constante renovación de las TIC (ver fig.2 Meta e-competencias).

En otras palabras, el desarrollo de habilidades de transacción relacionadas con crear, participar, colaborar, generar, innovar han de venir vinculadas al desarrollo de e-competencias, que desde una mirada más amplia podríamos calificar como una cultura digital.

\section{Conclusiones}

A continuación se recogen las tres preguntas expuestas al inicio de este trabajo, a fin de articular las conclusiones de este documento. ¿Cómo afectan las nuevas demandas del mundo del trabajo a las estrategias educativas actuales? ¿La incorporación de nuevas tecnologías en los entornos de aprendizaje ha resultado suficiente para preparar mejor a quienes se incorporan al mundo del trabajo? ¿La educación está cambiando más allá de la adopción de nuevos dispositivos tecnológicos?

A pesar que la idea de que vivimos en una sociedad basada en el uso intensivo del conocimiento no es nueva, aún queda mucho camino por recorrer. Tanto a nivel nacional como desde una perspectiva regional (en UE e igualmente en Iberoamérica) se requiere de más y mejores esfuerzos para preparar adecuadamente a las generaciones que habrán de atender los desafíos que esboza este nuevo siglo. No cabe duda que la irrupción de las tecnologías digitales ha contribuido a modificar (y en innumerables casos, a mejorar) muchas de las prácticas cotidianas. Sin embargo, la transformación que hoy se necesita no es únicamente de carácter técnico-instrumental, sino que por el contrario, ha de prestar especial atención a la formación constante de especialistas en la generación, administración y exportación del conocimiento.

En esta línea es fundamental lograr articular a los diferentes sectores de la sociedad a fin de consolidar una mirada transversal, holística pero al mismo tiempo representativa de los diferentes intereses y necesidades de cada región. En otras palabras, avanzar hacia un proyecto de sociedad basada en el uso intensivo del conocimiento cuyo principal valor agregado no esté en la calidad de los equipos tecnológicos que se utilicen ni en los índices de ancho de banda per capita, sino que en los planes estratégicos de educación, innovación y renovación del conocimiento.

De este modo, al lograr una adecuada articulación entre diferentes sectores de la sociedad, entonces podremos avanzar hacia modelos de desarrollo e innovación que resulten inclusivos y, al mismo tiempo, intensivos en el uso de conocimiento.

Probablemente, será necesario revisar y apuntalar las 
estrategias regionales tanto de la UE como de Iberoamérica para mermar las crecientes brechas y desencuentros que se observan entre las políticas de formación de recursos humanos altamente calificados y los sistemas educativos con que hoy se cuentan. Por ejemplo, es menester que la innovación no quede únicamente en la etapa de incorporación de nuevas tecnología a las escuelas y universidades, sino que estas estrategias y políticas vengan acompañadas de una verdadera transformación en los esquemas, modelos, ciclos y dinámicas de enseñanza y aprendizaje.

La década que empieza demanda repensar profundamente aquellos sistemas e instituciones educativas que no estén en condiciones de preparar una fuerza laboral capaz de reinventarse (y re-educarse) de manera continua y durante toda la vida. En esta línea, es probable que tras los esfuerzos de equipamiento tecnológico, ahora sea tiempo de prestar atención a aquellas fases de maduración tecnológica que ocurren cuando los dispositivos digitales dejan de ser el elemento diferenciador. Es decir, cuando las TIC se invisibilizan gracias a las etapas de maduración, domesticación, apropiación y de desarrollo de e-competencias. Ello ocurrirá cuando el componente diferenciador no esté en las tecnología per se sino en su uso y aprovechamiento. Es de esperarse, que éste entre otros aspectos esté en las agendas públicas y educativas de aquellos individuos tomadores de decisión que procuren un mejor devenir para nuestra sociedad.

La incorporación de tecnologías digitales en los entornos de aprendizaje ha venido a nutrir de nuevas herramientas, posibilidades y alcances a quienes están interesados en (des)aprender de manera continua. No hay dudas que un uso eficiente, oportuno y estratégico de las TIC puede convertirse en un potente insumo pedagógico y de aprendizaje continuo que trasciende de manera significativa los entornos formales de aprendizaje. Sin embargo, sería reduccionista apostar por las tecnologías digitales como la única fuente de aprendizaje. Hoy en día la evolución de las tecnologías da cuenta de la relevancia del componente social y de la importancia de aprender con y de otros. Por otra parte, tomando en consideración el componente tácito de los procesos de adquisición de saberes, habrá que recuperar (o revisibilIzar) aquellos espacios de aprendizaje basados en la conversación, la observación, la transferencia uno-a-uno, el aprender en la práctica y mediante el ensayo-error ya sea en un taller, oficina u otro lugar de interacciones cotidianas. No obstante, es oportuno admitir que cada vez más se expanden los contextos digitales de aprendizajes colectivos e individuales, así como las oportunidades que éstos ofrecen.

En relación a si la educación está cambiando más allá de la adopción de nuevas tecnologías, posiblemente la respuesta más fácil a esta pregunta sea simplemente decir que sí. Sin embargo, hay interrogantes latentes. ¿Han sido suficientes los cambios? ¿Las transformaciones son de forma o fondo?¿Cómo los sistemas educativos atienden a las demandas de una sociedad del conocimiento?¿Cuándo estos cambios se verán reflejados en la incorporación de nuevas formas de enseñanza y (des)aprendizaje?¿Existe el suficiente diálogo entre el mundo del trabajo y el mundo de la educación?¿̇Sólo bastará con tener más docentes e-competentes?¿Existen alternativas más integrales de transformación educativa que trasciendan la incorporación de nuevos dispositivos digitales? En fin, aunque no resulta eficiente responder una pregunta con nuevas interrogantes, ello da cuenta de aquellas dimensiones que aún quedan por explorar en futuros trabajos.
Por último, y advirtiendo que no hay que caer en la tentación de buscar fórmulas de éxito ni simplificadas, hay algunos criterios que parecen relevantes de ser tomados en consideración al pensar en políticas públicas orientadas a impulsar el uso estratégicos de las nuevas tecnologías:

\section{- Cambio de foco en las políticas públicas (de las TIC a la cultura digital). \\ - Cambio en la estrategia de implementación (de los gobiernos centrales a los gobiernos locales). \\ - Cambio integral (la TIC han incidido en la educación sólo cuando se generan mejoras transversales). Es decir, considerar la inclusión de las tecnologías digita- les dentro de un sistema más complejo de innovacio- nes pedagógicas. \\ - Desarrollar y generar estímulos para la formación de docentes e-competentes.}

Las cinco habilidades descritas son entendidas como competencias transversales, no excluyentes. Es decir, tomando en consideración los criterios aquí expuestos (ej.: Estrategias de Lisboa 2000-2010; Wagner: 2008; Silva: 2008; Siemens: 2004 y Gilster: 1997) es necesario avanzar hacia una estrategia integradora de las competencias, que trascienda con creces las habilidades y conocimientos aquí descritos.

Este trabajador e-competente ha de ser capaz de utilizar sus habilidades para incrementar sus niveles de empleabilidad.

Sin lugar a dudas, estas e-competencias trascienden con creces la utilización instrumental de las TIC. El uso de estas herramientas ha de orientarse a empoderar las capacidades intelectuales de generar, procesar y comunicar el conocimiento.

De no tomarse en consideración las tendencias aquí descritas, se corren al menos dos riesgos inminentes: 1) Se seguirá beneficiando a aquellos sectores que han tenido los medios para adquirir una formación de excelencia, excluyendo al resto de la población a trabajos mal remunerados o al desempleo y 2) Si no se encuentra una fuerza laboral con las competencias requeridas en el mercado del trabajo interno, la importación de capital humano calificado detonará profundas redefiniciones que sin duda pondrán en problemas a la fuerza de trabajo a nivel nacional.

Hoy no hay duda de la inminente necesidad de articular planes y estrategias conjuntas y de largo plazo, entre agentes de los sectores público, productivo-laboral y educativo.

Un desafío clave que ha de enfrentar la educación de nuestros días es poner énfasis en la transferibilidad y aplicabilidad de estas e-competencias en diferentes contextos. Es decir, un estudiante/trabajador e-competente en permanente estado de aprendizaje (y desaprendizaje), que aproveche tanto las instancias formales como las informales para actualizar sus saberes, pero por sobre todo capaz de adaptarse de manera continua a los cambios de un entorno cada vez más confuso, complejo e interdependiente.

\section{Bibliografía}

Buckingham, David (2007). Beyond technology: Children's Learning in the Age of Digital Culture. Cambridge: Wiley-Blackwell.

Bauman, Zygmunt (2008). Tiempos líquidos. Vivir en una época de incertidumbre. Ciudad de México: Tusquets/CONACULTA.

Bell, Daniel (1976). El advenimiento de la sociedad postindustrial. Madrid: Alianza. 
Cobo, Cristóbal (2009). Strategies to promote the development of e-competences in the next generation of professionals: European and International trends. SKOPE Issues Paper Series, 13, pp.1-100. http://www.cardiff.ac.uk/socsi/research/researchcentres/skope/publications/monographs/monograph13.pdf Fecha de consulta, 02.10.2009.

Comisión Económica para América Latina y el Caribe, [CEPAL] (2008) Espacios iberoamericanos: la economía del conocimiento. CEPAL. http://www.eclac.org/cgibin/getProd.asp?xml=/publicaciones/xml/9/34459/P 34459.xml\&xsl=/ddpe/tpl/pgf.xsl\&base=/tpl/top-bottom.xsl Fecha de consulta, 10.02.2010.

Drucker, Peter (1959). Landmarks of Tomorrow: A Report on the New 'Post-Modern' World. New York: Harper.

European Centre for the Development of Vocational Training [CEDEFOP] (2008b) Future skill needs in Europe: Medium-term forecast (Synthesis report). http://www.cedefop.europa.eu Fecha de consulta, 10.02.2010.

EUROSTAT (2006). How skilled are Europeans in using computers and the internet? Office for Official Publications of the European Communities. Issue 17. http://epp.eurostat.ec.europa.eu/cache/ITY_OFFPUB /KS-NP-06-017/EN/KS-NP-06-017-EN.PDF, Fecha de consulta, 19.02.2010.

Friedman, Thomas. (2005). The World is Flat: A Brief History of the Twenty-first Century. NY: Farrar Straus \& Giroux.

Gilster, Paul (1997). Digital Literacy. NY: John Wiley \& Sons, Inc.

Machlup, Fritz (1962). The Production and Distribution of Knowledge in the United States. Princeton: Princeton University Press.

OECD. (2006). Skills Upgrading, New Policy Perspectives. Paris: Organisation for Economic Co-Operation and Development.

Parlamento Europeo y Consejo de la Unión Europea (2006). Recomendación del Parlamento Europeo y El Consejo sobre las competencias clave para el aprendizaje permanente. Diario Oficial L 394 de 30.12.2006. http://eur-

lex.europa.eu/LexUriServ/LexUriServ.do?uri=OJ:L:2 006:394:0010:0018:ES:PDF. Fecha de consulta, 11.02.2010.

Siemens, George (2004). Conectivismo: Una teoría de aprendizaje para la era digital. Traducción: Diego E. Leal Fonseca.

http://www.diegoleal.org/social/blog/blogs/index.ph $\mathrm{p} / 2009 / 06 / 17 /$ stephen-downes-el-futuro-del-aprendizaje?blog=2 Fecha de consulta, 11.02.2010.
Silva, Elena (2008). Measuring skills for the 21st Century. Education Sector. http://www.educationsector.org/research/research_show.htm?doc_id=716323 Fecha de consulta, 11.02.2010.

Wagner, Tony (2008). The Global Achievement Gap. Philadelphia: Basic Books.

| Cita recomendada de este artículo

Cobo Romaní, Cristóbal (2010). Cultura digital y nuevos perfiles profesionales: desafíos regionales. @tic. revista d'innovació educativa. ( $n^{\circ}$ 5). URL. Fecha de consulta, dd/mm/aaaa. 